\title{
El Dilatómetro Sísmico SDMT para ensayos de suelos in situ
}

Fecha de entrega: 23 de abril 2012 Fecha de aceptación: 27 de febrero 2013

\section{Silvano Marchetti ${ }^{1}$, Diego Marchetti ${ }^{2}$ y Felipe Villalobos ${ }^{3}$}

${ }^{1}$ Dipartimento di Ingegneria Civile, Università dell'Aquila, Via Campo di Pile Zona industriale di Pile, 67100 L'Aquila, Italia, silvano@marchetti-dmt.it

${ }^{2}$ Studio Prof. Marchetti, Via Bracciano 38, 00189 Roma, Italia, diego@marchetti-dmt.it

${ }^{3}$ Laboratorio de GeoMateriales, Universidad Católica de la Santísima Concepción, Alonso de Ribera 2850, Casilla 297 Concepción, Chile, avillalobos@ucsc.cl

Este artículo describe el equipo de ensayo del dilatómetro plano DMT e importantes aplicaciones en Ingeniería Geotécnica. El ensayo DMT es cada vez más usado alrededor del mundo debido a que el equipo y método de ensayo son confiables, adaptables y robustos. Comparado con ensayos convencionales, el DMT ha probado ser de más bajo costo, menor tiempo de ejecución y mejor repetibilidad. El SDMT es un DMT con sensores sísmicos incorporados para medir la velocidad de ondas de corte $V_{S}$. Tanto el DMT como el SDMT pueden resultar particularmente útiles en proyectos donde la rigidez del suelo y las predicciones de asentamientos resultan críticas para un diseño adecuado. Además se discuten aplicaciones para detectar superficies de deslizamientos en taludes de arcilla, determinar curvas $P-y$ de pilotes cargados lateralmente, controlar el mejoramiento de suelos y compactación, evaluar el potencial de licuación $y$ determinar el módulo de Young secante para modelaciones numéricas.

Palabras clave: ensayos in situ DMT, SDMT, historia de tensiones, módulo de deformaciones
This article describes the flat dilatometer test equipment DMT and important applications in Geotechnical Engineering. The DMT test is more often used worldwide because the equipment and testing method are reliable, adaptable and robust. Compared with conventional tests, the DMT has proved to be of lower cost, short execution time and repeatability. The SDMT consists of a DMT with seismic sensors included in order to measure shear wave velocities $V_{S}$. The DMT and SDMT can be of particular usefulness in projects where soil stiffness and settlement assessment are key parameters for an appropriate design. Moreover, applications for slip surface detections in clay slopes, determination of $P-y$ curves in laterally loaded piles, soil improvement and compaction control, soil liquefaction potential assessment and the determination of the secant Young modulus for numerical modelling, are discussed.

Keywords: in situ tests DMT, SDMT, stress history, strain modulus

valor sin unidad ingenieril $\mathrm{N}$ (golpes/pie), se pierde el sentido real y físico cuando $\mathrm{N}$ es usado para correlacionar un gran espectro de parámetros geotécnicos de resistencia y rigidez del suelo. Esto puede resultar en grandes errores especialmente para valores bajos y altos de N. Por otro lado, Robertson (2012) afirma que de hecho el ensayo SPT, es muy caro considerando la baja calidad y baja cantidad de información directa que entrega por metro lineal. Si 
se toman en cuenta las variadas y mejores alternativas de equipos y técnicas de ensayo in situ que existen hoy en día, resulta conveniente conocer estas nuevas tecnologías. Una de estas alternativas es el equipo de ensayo dilatométrico DMT.

El Dilatómetro Plano DMT es un equipo para ensayos in situ desarrollado hace aproximadamente cuatro décadas (Marchetti, 1975, 1980). Su uso ha ido en aumento constante con el correr de los años y se emplea en prácticamente todos los países industrializados. El ensayo está estandarizado por la norma ASTM D6635 (2001, 2007) y el Eurocode 7 (1997, 2007). La Figura 1a muestra las partes que componen el equipo, la Figura $1 \mathrm{~b}$ muestra en detalle la paleta que es insertada en el suelo y la Figura 1c muestra un esquema de funcionamiento del equipo DMT.

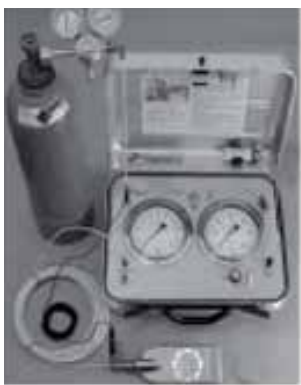

a)

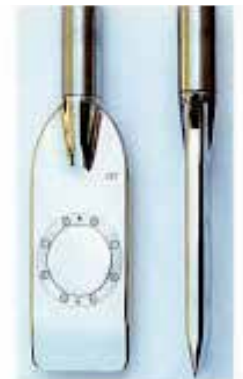

b)

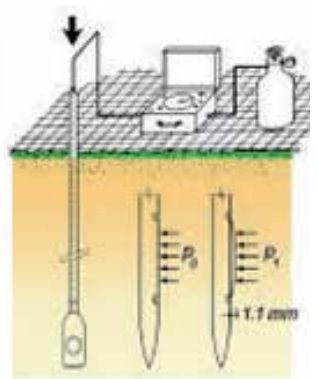

c)
Figura 1: Dilatómetro plano: a) partes del equipo, b) vista frontal mostrando membrana circular y vista lateral de la paleta del dilatómetro y c) diagrama del ensayo con el dilatómetro

Algunas de las características distintivas del DMT son:

- El DMT es un ensayo de penetración y como tal, tiene la gran ventaja de no requerir de un sondaje previo.

- El DMT, siendo un ensayo de carga lateral, proporciona información sobre la rigidez del suelo, información que no se puede obtener a partir de otros ensayos de penetración, los cuales esencialmente miden las características de "ruptura" del suelo, con lo cual se obtiene únicamente información sobre la resistencia en falla del suelo. Por otro lado, las distorsiones causadas por la sonda del DMT son apreciablemente menores que la alteración ocasionada por puntas cónicas (Baligh y Scott, 1975), ver figura 2.

- El equipo DMT es portátil, fácil de usar y excepcionalmente independiente del operador y repetible.

- El DMT proporciona un índice de la historia de tensiones. La historia de tensiones como tal no se utiliza en el diseño; sin embargo, su conocimiento es de sumo interés porque tiene una influencia dominante en el comportamiento del suelo.

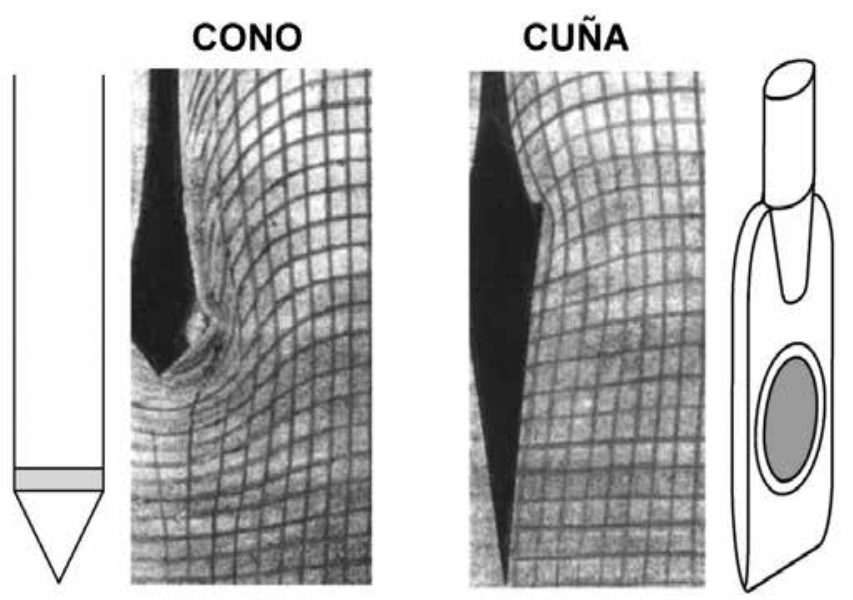

Figura 2: Distorsiones en un suelo cohesivo causada por la penetración de una sonda de forma cónica y una con forma de cuña (Baligh y Scott, 1975).

En el DMT se han incorporado sensores sísmicos denominándose SDMT. Esto permite la medición de la velocidad de ondas de corte $V_{S}$, además de la medición de los parámetros estáticos. Es importante hacer notar que $V_{S}$ se mide actualmente cada vez más por la necesidad de realizar análisis de respuesta sísmica de los suelos de fundación, para los cuales $V_{S}$ es un parámetro básico de entrada. En varios reglamentos sísmicos (Eurocode 8, 2004; BSSC, 2004; NCh 433 DS 61, 2011) se recomienda la determinación de $V_{S}$ en al menos los primeros $30 \mathrm{~m}$ de profundidad para proyectos de construcción ubicados en zonas sísmicas. El SDMT permite determinar la rigidez máxima $G_{0}$ bajo deformaciones pequeñas dado que de la elasticidad se tiene que el módulo de corte máximo es igual a $G_{0}=\rho V_{S}^{2}$, donde $\rho$ es la densidad del medio por donde se propagan las ondas de corte. Por otro lado, la rigidez para deformaciones en condiciones de servicio puede ser representada por el módulo edométrico $M_{D M T}$. Estos dos valores de rigidez pueden servir de orientación al seleccionar las curvas de degradación $G-\gamma$, es decir, la disminución del módulo de corte $G$ en función de la deformación de corte $\gamma$. Amoroso et al. $(2012 \mathrm{a}, \mathrm{b})$ presentan esta metodología y definen rangos para los cuales es posible intersectar los datos medidos de $G_{0}$ y $M_{D M T}$ en las curvas de degradación previamente disponibles (Figura 3). 


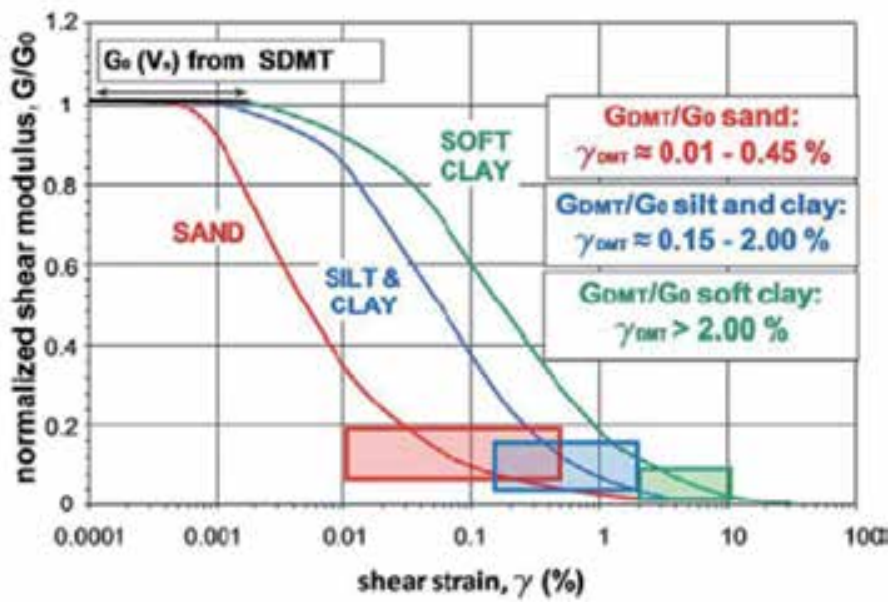

Figura 3: Ejemplos de curvas de degradación de rigidez y rangos de deformación de corte para arenas, limos y arcillas y arcillas blandas (Amoroso et al., 2012a)

\section{Ensayos con el dilatómetro DMT}

El dilatómetro plano está formado por una paleta de acero que contiene una delgada membrana circular expandible de acero montada en una de sus caras. Estando en reposo, la membrana queda al ras con la superficie plana de la paleta que la rodea. La paleta está conectada mediante una manguera electro-neumática que corre a lo largo de las barras de hincado, a una caja de control en la superficie (ver Figura 1a).

La unidad de control está equipada con manómetros, una señal audiovisual, una válvula de regulación de la presión del gas (proporcionado por un tanque) y válvulas de escape. La paleta se puede introducir en el terreno usando equipos de sondaje convencionales, ya sea por penetración estática como en el ensayo de penetración de cono CPT o por penetración dinámica/percusión con equipos de perforación usados en ensayos SPT convencional. El ensayo se inicia introduciendo la paleta del dilatómetro verticalmente en el terreno. Cuando la paleta ha alcanzado la profundidad deseada se suspende la penetración. El suelo presiona la membrana contra la paleta, lo cual emite una señal acústica en la superficie. Entonces, el operador infla la membrana y toma, en un lapso de 30 s dos lecturas: la presión $A$, necesaria para justamente empezar a desplazar la membrana (presión de 'despegue') y la presión $B$, requerida para empujar el centro de la membrana una distancia de $1.1 \mathrm{~mm}$ contra el suelo. Se puede también tomar opcionalmente una tercera lectura
$C$ (presión de cierre) al desinflar lentamente la membrana justamente después de alcanzar la presión $B$ y regresar la paleta a su posición original. Se continúa hincando la paleta hasta alcanzar la siguiente profundidad de ensayo, en incrementos típicos de avance cada $20 \mathrm{~cm}$.

Las lecturas de presión $A$ y $B$ obtenidas en el ensayo se deben corregir para tomar en cuenta el efecto de la rigidez de la membrana, la cual puede ser importante especialmente en suelos sueltos y blandos. Estos aspectos de corrección pueden ser consultados en TC16 (2001). En primer lugar las lecturas de terreno corregidas se convierten en los parámetros intermedios del DMT conocidos como índice del material $I_{D}$, índice de tensión horizontal $K_{D}$ y módulo del dilatómetro $E_{D}$. Luego $I_{D}, K_{D}$ y $E_{D}$ se convierten mediante correlaciones de uso común, en: módulo edométrico $M$, resistencia al corte no drenada $s_{u}$, coeficiente de empuje en reposo $K_{0}$ (arcillas), OCR (arcillas), ángulo de fricción interna $\phi$ ' (arenas) y peso unitario $\gamma$. Se pueden estimar los coeficientes de consolidación $C_{h}$ y de permeabilidad $k_{h}$ mediante la realización de ensayos de disipación (Totani et al., 1998).

Ejemplos de perfiles obtenidos con el DMT se muestran en la Figura 4. El índice del material $I_{D}$ proporciona información sobre el tipo de suelo (arena, limo, arcilla).

$I_{D}=\frac{\mathrm{p}_{1}-\mathrm{p}_{0}}{\mathrm{p}_{0}-\mathrm{u}_{0}}$

Donde $\mathrm{u}_{0}$ es la presión de poros hidrostática. La Figura $4 \mathrm{a}$ muestra que hasta los $4 \mathrm{~m}$ hay una transición de arena, limo y arcilla, hasta los $20 \mathrm{~m}$ existe una arcilla con lentes de limo a los $16 \mathrm{~m}$, luego hasta los $26 \mathrm{~m}$ hay principalmente limo y finalmente hay arcilla hasta los $36 \mathrm{~m}$.

El índice de tensión horizontal $K_{D}$ es interpretado como un coeficiente de empuje lateral en reposo amplificado por la penetración de la paleta.

$K_{D}=\frac{\mathrm{p}_{0}-\mathrm{u}_{0}}{\sigma_{\mathrm{v} 0}^{\prime}}$

Donde $\sigma_{\mathrm{v} 0}^{\prime}$ es la tensión vertical efectiva. La variación de $K_{D}$ con la profundidad es similar en forma al perfil de la relación de sobreconsolidación $O C R$. Un valor $K_{D} \approx 2$ equivale a suelos normalmente consolidados $\mathrm{NC}$, es decir, $O C R=1 ; K_{D}>2$ indica preconsolidación. Es por ello que 
el perfil de $K_{D}$ puede ser usado como un indicador de la historia de tensiones del depósito. Del ejemplo de la Figura $4 \mathrm{~d}$ se tiene que el suelo es $\mathrm{NC}$.

El módulo del dilatómetro $E_{D}$ es obtenido por medio de la teoría de la elasticidad, la geometría de la membrana y el desplazamiento de $1.1 \mathrm{~mm}$ como (Marchetti, 1980),

$$
E_{D}=\frac{E}{1-v^{2}}=34.7\left(\mathrm{p}_{1}-\mathrm{p}_{0}\right)
$$

donde $v$ es la razón de Poisson y $E$ es el módulo de Young. En la Figura 4b se muestra la variación con la profundidad del módulo edométrico $M$ vertical, drenado y bajo tensión geostática. $M$ se determina de $E_{D}$ usando un factor que es función de $K_{D}$ (Marchetti, 1980). La Figura 4c muestra la variación de la resistencia al corte no drenada $s_{u}$, la cual se observa aumenta de forma casi lineal con la profundidad.

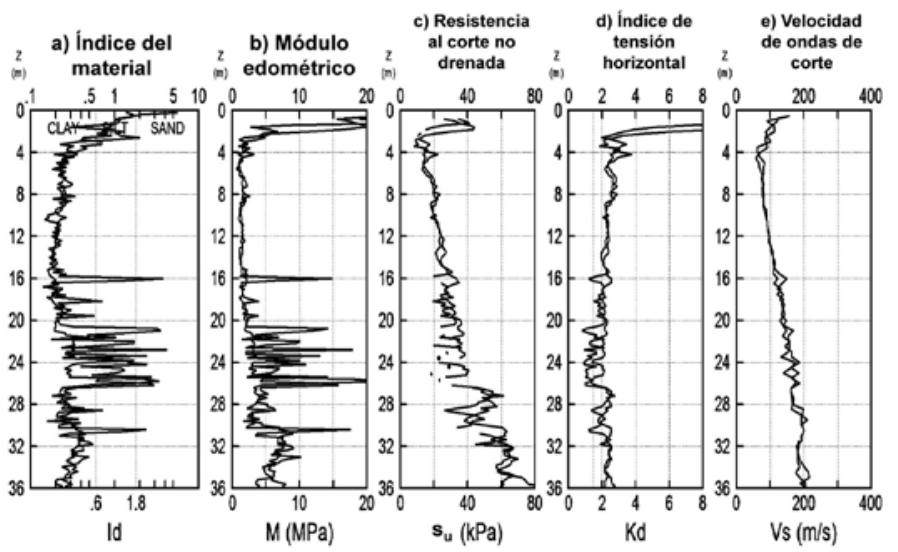

Figura 4: Ejemplos de resultados de dos sondajes próximos en Fiumicino, Italia, mostrando la variación con la profundidad de a) $I_{D}$, b) $M$, c) $s_{u}$, d) $K_{D}$ y e) $V_{S}$

Información detallada sobre del equipo DMT, el procedimiento de ensayo y las fórmulas de interpretación se puede consultar en el amplio informe preparado por el comité técnico ISSMGE TC16 (2001).

\section{Ensayos con el dilatómetro sísmico SDMT}

Después de haber sido introducido por primera vez por Hepton (1988), el SDMT fue mejorado en Italia (Marchetti et al., 2008; Monaco et al., 2009). El SDMT incluye en el dilatómetro plano sensores sísmicos para la medición de la velocidad de ondas de corte. La Figura 5a muestra un elemento cilíndrico ensamblado a la paleta DMT, el cual está equipado con dos receptores localizados a 0.5 $\mathrm{m}$ de distancia. Cuando se genera una onda de corte en la superficie, llega primero al receptor superior (azul) y luego, transcurrido un retraso, al receptor inferior (rojo). La velocidad $V_{s}$ se obtiene como la relación de diferencia de distancias entre la fuente y los dos receptores $\left(\mathrm{S}_{2}\right.$ $\mathrm{S}_{1}$ ) y el retraso $\Delta t$ entre el primer receptor y el segundo (Figura 5b). Los sismogramas generados por los dos receptores, una vez amplificados y digitalizados en función de la profundidad, se transmiten a un computador en la superficie, que determina el retraso $\Delta t$ (Figura $5 \mathrm{c}$ ). Utilizar dos receptores evita la posible imprecisión en la determinación del "tiempo cero" al momento del impacto del martillo que a veces se observa en la configuración con un sólo receptor dentro del pseudointervalo. Por otro lado, el par de sismogramas registrado por los dos receptores para una cierta profundidad de prueba corresponde al mismo impacto del martillo y no a diferentes golpes en serie, no necesariamente idénticos. De ahí que la repetibilidad de las mediciones de $V_{s}$ mejora considerablemente, siendo la repetibilidad observada de $V_{s} \approx 1 \%$, es decir, unos cuantos $\mathrm{m} / \mathrm{s}$ (Totani et al., 2009). Las mediciones de $V_{s}$ se toman cada $0.5 \mathrm{~m}$ de profundidad.

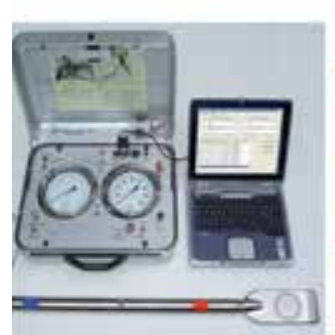

a)

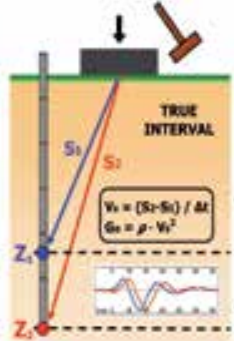

b)

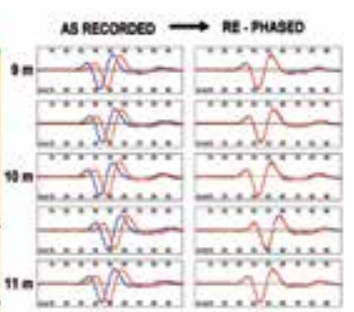

c)
Figura 5: Dilatómetro sísmico, a) unidad de control, computador y paleta del DMT con sensores sísmicos acoplados, b) esquema del ensayo sísmico con el dilatómetro y c) ejemplo de registros de señales en tres profundidades en Fucino, Italia

La fuente de ondas de corte en la superficie es un martillo de péndulo $(\approx 10 \mathrm{~kg})$ que golpea horizontalmente a una base en forma de paralelepípedo empujada verticalmente contra el suelo (por ejemplo con el peso de un camión) y orientada con su eje mayor paralelo al eje de los receptores de tal forma que puedan ofrecer la sensibilidad más alta a la onda de corte.

La Figura 4e ilustra un ejemplo de distribución de $V_{s}$ con la profundidad $z$ obtenido con el SDMT, donde se aprecia un incremento casi lineal con $z$. 


\section{Tipos de suelos a ensayar}

Los suelos que se pueden investigar mediante el equipo DMT varían entre suelos extremadamente blandos, suelos duros y rocas blandas. El DMT resulta adecuado para arenas, limos y arcillas, cuyas partículas son pequeñas comparadas con el diámetro de la membrana $(60 \mathrm{~mm})$. No se recomienda para grava y roca. Sin embargo, la paleta es lo suficientemente robusta para atravesar capas de grava con un espesor del orden de $0.5 \mathrm{~m}$. Las lecturas del DMT son muy precisas incluso en suelos de consistencia prácticamente líquida. Por otro lado, la paleta es muy robusta, puede soportar con seguridad fuerzas de empuje de hasta $250 \mathrm{kN}$ e incluso puede penetrar roca blanda. Las arcillas se pueden ensayar entre valores de $s_{u}$ de 2 a $4 \mathrm{kPa}$ hasta valores de $1000 \mathrm{kPa}$ (margas). El intervalo de variación del módulo $M$ medible oscila entre $0.4 \mathrm{MPa}$ y $400 \mathrm{MPa}(\mathrm{TC} 16,2001)$.

\section{Aplicaciones a problemas de ingeniería Asentamiento de fundaciones superficiales}

La estimación de asentamientos de fundaciones superficiales ha sido una de las aplicaciones más útiles del DMT (Schnaid, 2009), sobre todo en arenas en las que no se pueden recuperar muestras inalteradas. El asentamiento se calcula generalmente mediante la fórmula unidimensional,

$$
S_{1-D M T}=\sum_{i=1}^{n} \frac{\Delta \sigma_{v i}}{M_{D M T i}} \Delta z
$$

determinando la variación del incremento de carga $\Delta \sigma_{v}$ con el incremento de profundidad $\Delta z$ de acuerdo con la formulación de Boussinesq que asume al suelo como un semi-espacio elástico e infinito, donde el cálculo se discretiza para las $n$ mediciones de $M_{D M T}$ La Figura 6 muestra un esquema de variación del módulo edométrico obtenido con el DMT con la profundidad, además de la variación del incremento de tensiones $\Delta \sigma_{\mathrm{v}}$ con la profundidad debido a la sobrecarga de un terraplén para así estimar el asentamiento $S$.

Notar que el asentamiento calculado con la expresión (4) representa condiciones de servicio, es decir, para un factor de seguridad FS entre 2.5 y 3.5. Esto es porque el $M_{D M T}$ ha sido correlacionado con módulos determinados de retro análisis para zapatas y losas de fundación. También se puede notar que el asentamiento estimado de esta manera se puede interpretar preliminarmente en arcillas como un asentamiento por consolidación primaria, si $M_{D M T}$ ha sido derivado de la curva edométrica para el rango de incremento de tensiones adecuado (TC16, 2001).

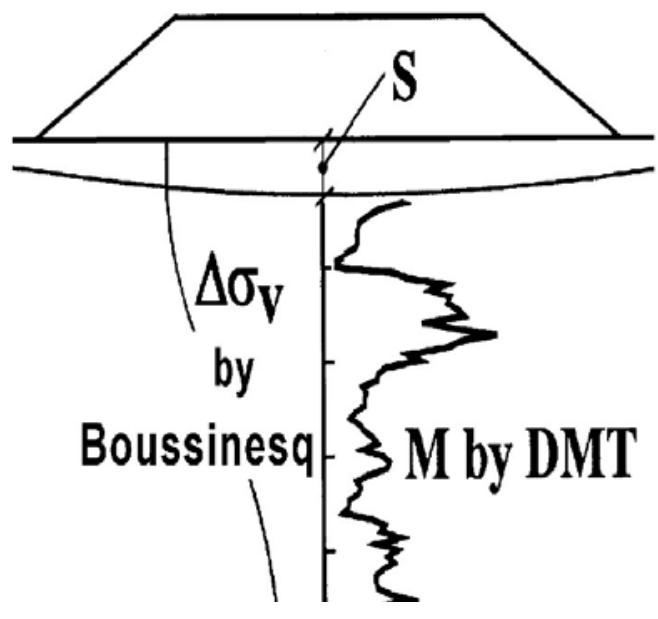

Figura 6: Esquema de la variación de $M_{D M T}$ y $\Delta \sigma_{\mathrm{v}}$ con $z$ para la estimación del asentamiento $S$ debido a la carga de un terraplén

La validez del método ha sido confirmada por un gran número de concordancias observadas entre los asentamientos medidos y los calculados con DMT. Schnaid (2009) presenta 16 casos donde el promedio del valor estimado usando el DMT dividido por el valor medido es de 1.18 con una desviación estándar de 0.38 . La buena capacidad de predicción del DMT se considera que se debe a que: (a) la penetración del suelo con una cuña distorsiona menos el suelo que con un cono (Figura 2); (b) el módulo obtenido en un ensayo de minicarga (expansión de la membrana) está inherentemente mucho más relacionado con el módulo del suelo que la resistencia a la penetración (falla del suelo).

\section{Desplazamiento de pilotes cargados lateralmente}

Los resultados de sondajes con DMT permiten trazar las curvas $P-y$, donde $P$ es la carga horizontal sobre el pilote e $y$ es el desplazamiento horizontal del pilote. Robertson et al. (1987) proponen un método de cálculo para arcillas y arenas que se basa en los parámetros $E_{D}$, $\phi$ ' y $K_{0}$ que se pueden determinar mediante el DMT y una expresión adimensional parábolica entre la carga y la deflexión horizontal. Marchetti et al. (1991) proponen otro método de cálculo para arcillas que se basa en un cálculo directo 
mediante una expresión adimensional hiperbólica. Los dos métodos generan predicciones de desplazamiento horizontal semejantes y son muy sensibles a la rigidez adoptada. Tomar en cuenta que estos métodos se aplican al caso de carga monotónica aplicada por primera vez.

\section{Superficies de deslizamiento en arcillas OC}

Totani et al. (1997) desarrolló un método rápido para detectar superficies de deslizamiento activas o antiguas en taludes de arcilla preconsolidada OC, tomando como base la inspección de los perfiles del indicador $K_{D}$. Básicamente, el método consiste en identificar zonas de arcilla normalmente consolidada $\mathrm{NC}$ en un talud que, de otra manera, exhibe un perfil OC. Los estratos de arcilla $\mathrm{NC}$, remoldeados por deslizamientos anteriores y luego reconsolidados bajo el peso del suelo sobreyacente, se pueden identificar aplicando un valor $K_{D} \approx 2$ como identificador de las zonas $\mathrm{NC}$, como se esquematiza en la Figura 7.
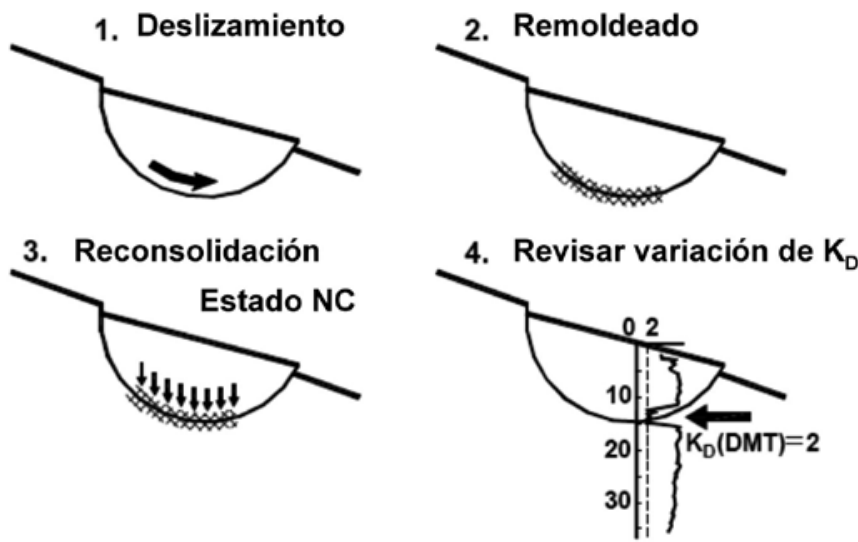

Figura 7: Detección de superficies de deslizamiento en taludes de arcilla $\mathrm{OC}$ usando el parámetro $K_{D}$ obtenido en ensayos con DMT (de Totani et al., 1997)

\section{Control de mejoramiento de suelos}

Para controlar el mejoramiento de suelos se pueden realizar sondajes con el DMT antes, durante y después del mejoramiento. Schmertmann et al. (1986) indican que el aumento porcentual en $M_{D M T}$ es de aproximadamente el doble del incremento de la resistencia de punta del cono $q_{t}$, en el ensayo CPT. En otras palabras, el $M_{D M T}$ es más sensible y aumenta con mayor rapidez a los cambios de rigidez del suelo que $q_{t}$. El método DMT es por lo tanto adecuado para detectar variaciones pequeñas en el esfuerzo horizontal, por ejemplo, en el suelo sometido a relajación de tensiones detrás de muros pantalla durante la excavación (Monaco y Marchetti, 2004).

\section{Control de compactación}

El ensayo DMT puede también ser usado como una útil herramienta para el control de la compactación de la subrasante sobre la que se apoyarán subbases, bases y el pavimento de una carretera (Marchetti, 1994). El perfil de aceptación $M_{D M T}$ se puede establecer al ejecutar unos cuantos ensayos preliminares DMT sobre la subrasante aceptada, tomando como base los métodos originalmente especificados, tales como Proctor, razón de soporte de California CBR o ensayos de placa de carga. Luego, trazar un perfil $M_{D M T}$ promedio. El perfil de diseño $M_{D M T}$ podrá entonces usarse como un método económico para el control rutinario de calidad de la compactación.

Una tendencia en el diseño actual de pavimentos es usar los módulos en lugar del CBR o la densidad seca máxima compactada DSMC del Proctor. Por lo tanto, los perfiles de $M_{D M T}$ podrán proporcionar información alternativa y útil al diseñador. El uso de métodos de medición de rigidez, alternativos al Proctor y CBR, adquieren relevancia en casos en que simplemente no se pueda obtener la humedad óptima del ensayo Proctor en el terreno, por ejemplo, en lugares desérticos o muy húmedos a saturados.

\section{Evaluación del potencial de licuación}

En la Figura 8a se muestra un gráfico que proporciona estimaciones de la Relación de Resistencia Cíclica $C R R$ de una arena limpia $(\mathrm{CF}<5 \%)$ mediante el uso del DMT basada en $K_{D}$. Mientras que la Figura 8b muestra que con el SDMT se obtiene una segunda estimación independiente de $C R R$, basada en $V_{s}$. Para sismos de magnitud 7.5, la curva para estimar $C R R$ a partir de $K_{D}$ es la propuesta por Monaco et al. (2005),

$C R R_{7.5}=0.0107 K_{D}^{3}-0.0741 K_{D}^{2}+0.2169 K_{D}-0.1306$

y para estimar $C R R$ a partir de $V_{S}$ es el gráfico de Andrus y Stokoe (2000),

$C R R_{7.5}=\left[0.022\left(\frac{K_{a 1} V_{S 1}}{100}\right)^{2}+2.8\left(\frac{1}{V_{S 1}^{*}-K_{a 1} V_{S 1}}-\frac{1}{V_{S 1}^{*}}\right)\right] K_{a 2}$

donde $V_{S I}$ es la velocidad de ondas de corte estandarizada para una sobrecarga de $1 \mathrm{~kg} / \mathrm{cm}^{2}$ (presión atmosférica $p_{a}$ de 
$100 \mathrm{kPa})$.

$$
V_{S 1}=V_{S}\left(\frac{p_{a}}{\sigma_{v 0}^{\prime}}\right)^{0.25}
$$

$\sigma_{v 0}$ es la tensión vertical efectiva inicial, $V_{S 1}^{*}$ es el valor máximo de $V_{S I}$ para que ocurra licuación (de acuerdo a la Figura $8 \mathrm{~b} V_{S 1}^{*} \approx 200 \mathrm{~m} / \mathrm{s}$ para suelos con contenidos de finos de $20 \%$ ) y $K_{a 1}$ es un factor para corregir $V_{S 1}$ y $K_{a 2}$ para corregir $C R R$ cuando existen suelos cementados por efecto de envejecimiento. Tanto $K_{a l}$ como $K_{a 2}$ son iguales a 1.0 cuando los suelos son no cementados del Holoceno (12000 años de edad hasta el presente).

a)

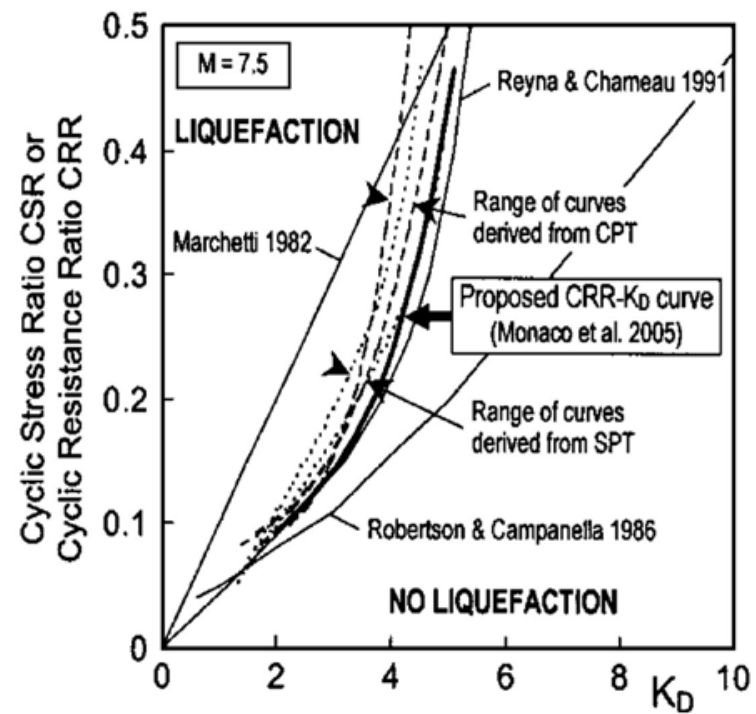

b)

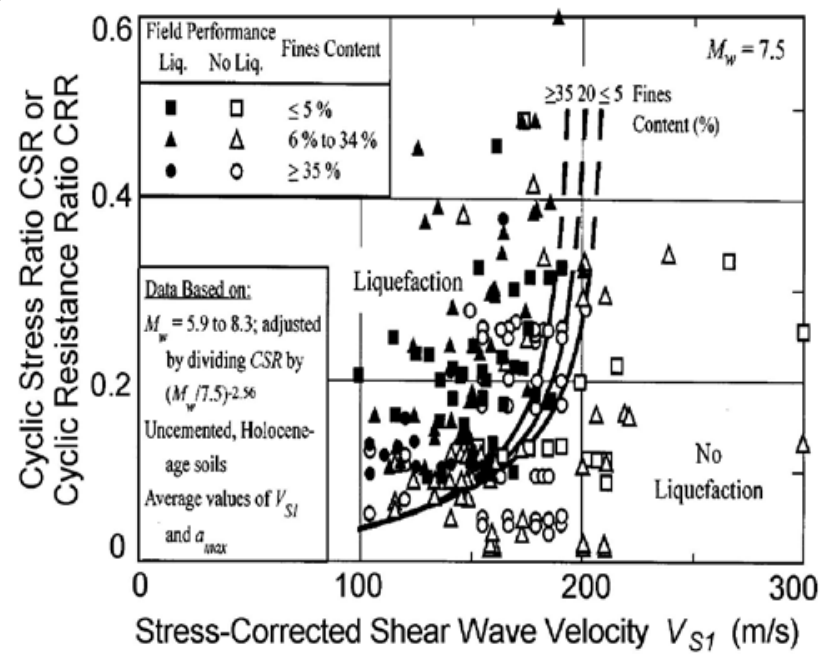

Figura 8: Curvas para evaluar CRR en arenas limpias, a) en función de $K_{D}$ (Monaco et al., 2005) y b) en función de $V_{S}$ (Andrus y Stokoe, 2000)

Datos experimentales (Marchetti, 2010), y un amplio programa comparativo con estanques de ensayo de calibración en la Universidad de Corea (Lee et al., 2010) han mostrado que $K_{D}$ es considerablemente más sensible que $q_{t}$ respecto a la historia de tensiones (incluyendo el envejecimiento). Por el contrario, $q_{t}$ es casi independendiente del estado de deformaciones histórico a lo largo de la línea $K_{0}$ (Baldi et al., 1985) y no es muy sensible a la historia de tensiones (Schnaid, 2009). Sin embargo, la historia de tensiones es un importante factor en el fenómeno de licuación debido a su apreciable influencia sobre el valor de CRR. Baldi et al. (1985) señalan que para obtener estimaciones confiables de la susceptibilidad de las arenas a licuar, se requiere de equipos de ensayo (distintos al CPT y SPT) más sensibles que detecten los efectos relacionados a la historia de tensión-deformación del suelo. Debido a que la historia de tensiones es un parámetro fundamental para la determinación del $C R R$, no es de extrañar que, en ausencia de ésta, la estimación de $C R R$ a partir del ensayo CPT resulte en una amplia dispersión. La mayor sensibilidad de $K_{D}$ a la historia de tensiones sugiere que $K_{D}$ pudiera tener una relación más estrecha con $C R R$ que la existente para $q_{t}$.

Yu (2004) ha identificado la relación entre $K_{D}$ y el parámetro de estado $\psi$, correspondiente a la distancia vertical entre la línea de estado actual y la de estado crítico en la presentación convencional $e-\ln p$ ' para la misma tensión media efectiva $p$ '. El parámetro $\psi$ gobierna la tendencia de una arena a aumentar o disminuir de volumen ante tensiones de corte, por lo que $\psi$ está íntimamente relacionado con la resistencia a la licuación $C R R$. Por lo tanto, la relación $K_{D}-\psi$ constituye otro elemento en el que se apoya la probabilidad de una buena relación $K_{D}-C R R$. De hecho, a pesar de la fuerte relación $\psi$ - $C R R$, incluso $\psi$ es un indicador imperfecto de $C R R$, ya que $\psi$ es insensible a la historia de tensiones mientras que $C R R$ aumenta con la historia de tensiones. No parecerá entonces ilógico esperar que $K_{D}$, siendo un parámetro relacionado con $\psi$, pero al mismo tiempo relacionado también con la historia de tensiones, la cual no existe para $\psi$, pudiera estar exclusivamente bien correlacionada con $C R R$.

Según se indicó anteriormente, el SDMT también proporciona una segunda estimación independiente de $C R R$, la cual se puede obtener a partir de $V_{s}$. En caso de diferencias entre las dos estimaciones de $C R R$, 
generalmente se le asigna un mayor valor a $C R R\left(K_{D}\right)$, debido a que $V_{s}$ es más bien insensible a la historia de tensiones (Monaco y Marchetti, 2007). Además, $V_{s}$ es una medición correspondiente a una deformación muy pequeña y se correlaciona desfavorablemente con un fenómeno de deformaciones mucho más grandes como es el caso de la licuación.

\section{Uso del DMT para modelación con MEF}

La forma más simple estriba en adoptar el modelo elástico lineal, en el cual el módulo de Young $E$ se calcula con la aproximación $E \approx 0.8 M_{D M T}$ (Hamza y Richards, 1995).

La buena capacidad de predicción de asentamientos a partir de los resultados del DMT se puede usar para verificar resultados usando programas computacionales con el Método de Elementos Finitos MEF. Una forma de obtener esa verificación consiste en ejecutar un ensayo DMT y así obtener un perfil de $M_{D M T}$ para predecir el asentamiento para un caso simple de carga, por ejemplo, bajo un estanque circular uniformemente cargado que transmite una carga de $100 \mathrm{kPa}$. Luego, realizar la misma predicción mediante el programa de cálculo MEF. Si los dos asentamientos calculados son muy diferentes, tal vez haga falta ajustar los parámetros MEF. La idea es usar el ensayo DMT en sustitución de una prueba de carga in situ.

Al ocupar el modelo no lineal HS (suelo duro) en Plaxis, el dato de entrada básico recomendado es el módulo de Young secante $E_{50}^{\text {ref }}$, el cual es calculado a partir de ensayos triaxiales normalizados (referenciados) a $100 \mathrm{kPa}$ para el $50 \%$ de la tensión desviadora máxima. Schanz y Vermeer (1997) han demostrado que $E_{50}^{\text {ref }}$ se correlaciona con $M$ y que, para muchas arenas cuarzosas, el valor de $E_{50}^{r e f}$ se encuentra dentro del intervalo de variación de 15 a $75 \mathrm{MPa}$. Este intervalo es sorprendentemente similar al intervalo encontrado para el módulo $M_{D M T}$ en varias arenas. Por lo tanto, existe la alternativa de determinar $M_{D M T}$ en ensayos DMT como valores de $E_{50}^{\text {ref }}$ a usar en Plaxis, dada la proximidad que existe entre ellos. Monaco y Marchetti (2004) presentan aplicaciones numéricas usando Plaxis y $E_{50}^{\text {ref }}$ en el estudio de muros pantalla con varios niveles de arriostramiento.

\section{Conclusiones}

El Dilatómetro de Marchetti DMT es un equipo de exploración de suelos versátil y de gran apoyo para el Ingeniero Geotécnico en la obtención de valores de parámetros de diseño adecuados. Representa una alternativa más rápida y económica a los ensayos de laboratorio y a varios de los ensayos in situ actualmente en uso. Entre sus ventajas principales destacan la portabilidad, la sencillez de su operación, la variedad de maneras de penetrar la paleta así como de los tipos de suelos posibles de explorar. La incorporación de sensores sísmicos en el DMT permite obtener perfiles de $V_{s}$ además de $s_{u}, M$, OCR, $K_{0}$ y $\phi$ en un mismo sondaje. Las correlaciones y los procedimientos utilizados están ampliamente documentados y cuentan con el respaldo de la norma ASTM D6635 (2007) y del Eurocode 7 (2007). El uso del DMT entrega al Ingeniero Geotécnico mayores opciones de análisis, como las que han sido presentadas en este artículo.

\section{Referencias}

Amoroso, S., Monaco, P. and Marchetti, D. (2012a). Use of the Seismic Dilatometer (SDMT) to estimate in situ $G-\gamma$ decay curves in various soil types. Proceedings of the International Conference on Geotechnical and Geophysical Site Characterization 4, Coutinho \& Mayne (eds), Taylor \& Francis, Vol. 1, 489 -497.

Amoroso, S., Lehane, B.M. and Fahey, M. (2012b). Determining $G-\gamma$ decay curves in sand from a Seismic Dilatometer Test (SDMT). Proceedings of the International Conference on Geotechnical and Geophysical Site Characterization 4, Coutinho \& Mayne (eds), Taylor \& Francis, Vol. 1, 447 -452.

Andrus, R.D. and Stokoe II, K.H. (2000). Liquefaction resistance of soils from shear-wave velocity. Journal of Geotechnical and Geoenvironmental Engineering 126, No.11, 1015-1025.

ASTM D6635 (2001, 2007). Standard Test Method for Performing the Flat Plate Dilatometer. Book of Standards, West Conshohocken, PA, USA.

Baldi, G., Bellotti, R., Ghionna, V., Jamiolkowski, M. and Pasqualini, E. (1985). Penetration resistance and liquefaction of sands. Proceedings of the $11^{\text {th }}$ ICSMFE, San Francisco, vol. 4, 1891-1896.

Baligh, M.M. and Scott, R.F. (1975). Quasi Static Deep Penetration in Clays. Journal of the Geotechnical Engineering Division 101, No. 11, 1119-1133.

BSSC Building Seismic Safety Council (2004). NEHRP Recommended provisions for seismic regulations for new 
buildings and other structures. FEMA 450/451, 19-38.

Eurocode 7 (1997, 2007). Geotechnical Design - Part 2: Ground Investigation and Testing. EN 1997-2:2007.

Eurocode 8 (2004). Design of structures for earthquake resistance. Part 1: general rules, seismic actions and rules for buildings. EN 1998-1: 2004.

Hamza, M. and Richards, D.P. (1995). Correlations of DMT, CPT and SPT in Nile Basin Sediment. Proceedings $11^{\text {th }}$ African Conference SMFE, Cairo Egypt, 437-446.

Hepton, P. (1988). Shear wave velocity measurements during penetration testing. Proceedings of Penetration Testing in the UK, ICE, 275-278.

Lee, M.J., Choi, S.K., Kim, M.T. and Lee, W. (2011). Effect of stress history on CPT and DMT results in sand. Engineering Geology 117 (3-4), 259-265.

Marchetti, S. (2010). Sensitivity of CPT and DMT to stress history and aging in sands for liquefaction assessment. Proceedings CPT 2010 International Symposium Huntington Beach, California.

Marchetti, S., Monaco, P., Totani, G. and Marchetti, D. (2008). In situ tests by seismic dilatometer (SDMT). Proceedings From Research to Practice in Geotechnical Engineering, ASCE Geotech. Spec. Publ. No. 180 (honoring J.H. Schmertmann), 292-311.

Marchetti, S. (1994). An example of use of DMT as an help for evaluating compaction of subgrade and underlying embankment. Internal Technical Note.

Marchetti, S., Totani, G., Calabrese, M. and Monaco, P. (1991). $\mathrm{P}-\mathrm{y}$ curves from DMT data for piles driven in clay. Proceedings of the $4^{\text {th }}$ International Conference on Piling and Deep Foundations, DFI, Stresa, Vol. 1, 263-272.

Marchetti, S. (1982). Detection of liquefiable sand layers by means of quasi-static penetration tests. Proceedings $2 \mathrm{nd}$ European Symposium on Penetration Testing, Amsterdam, vol. 2, 689-695.

Marchetti, S. (1980). In Situ Tests by Flat Dilatometer. Journal of the Geotechnical Engineering Division 106, No. 3, 299-321.

Marchetti, S. (1975). A New in Situ Test for the Measurement of Horizontal Soil Deformability. Proceedings of the Conference on In Situ Measurement of Soil Properties, ASCE Spec. Conf.,
Raleigh, Vol. 2, 255-259.

Mayne, P.W., Coop, M.R., Springman, S.M., Huang, A.B. and Zornberg, J.G. (2009). Geomaterial behaviour and testing. State of the art Paper. Proceedings of the $17^{\text {th }}$ ICSMGE, Alexandria, Egypt, Vol. 4: 2777-2872.

Monaco, P., Marchetti, S., Totani, G. and Marchetti, D. (2009). Interrelationship between small strain modulus $\mathrm{G}_{0}$ and operative modulus. Proceedings of the International Conference on Performance-Based Design in Earthquake Geotechnical Engineering, Kokusho, Tsukamoto and Yoshimine (eds.), Tsukuba, Taylor \& Francis, 1315-1323.

Monaco, P. and Marchetti, S. (2007). Evaluating liquefaction potential by seismic dilatometer (SDMT) accounting for aging/ stress history. Proceedings of the $4^{\text {th }}$ International Conference on Earthquake Geotechnical Engineering ICEGE4, Thessaloniki, paper 1626.

Monaco, P., Marchetti, S., Totani, G. and Calabrese, M. (2005). Sand liquefiability assessment by Flat Dilatometer Test (DMT). Proceedings XVI ICSMGE, Osaka, vol. 4: 2693-2697.

Monaco, P. and Marchetti, S. (2004). Evaluation of the coefficient of subgrade reaction for design of multi-propped diaphragm walls from DMT moduli. Proceedings International Site Characterization ISC'2, Porto, Portugal.

NCh 433 DS 61 (2011). Diseño sísmico de edificios. Decreto 61. Reglamento que fija el diseño sísmico de edificios. Santiago, Chile.

Reyna, F. and Chameau, J.L. (1991). Dilatometer based liquefaction potential of sites in the Imperial Valley. Proceedings 2nd International Conference on Recent Advances in Geotechnical Earthquake Engineering and Soil Dynamics, St. Louis, 385-392.

Robertson, P.K. (2012). Interpretation of in-situ tests - some insights. The James K. Mitchell lecture. Proceedings of the International Conference on Geotechnical and Geophysical Site Characterization 4, Porto de Galinhas, Brasil, Coutinho \& Mayne (eds.), Taylor \& Francis, Vol. 1, 3 -24.

Robertson, P.K. and Campanella, R.G. (1986). Estimating liquefaction potential of sands using the Flat Plate Dilatometer. Geotechnical Testing Journal 9(1), 38-40.

Robertson, P.K., Davies, M.P. and Campanella, R.G. (1987). Design of laterally loaded driven piles using the flat dilatometer. 
Geotechnical Testing Journal 12, No. 1, 30-38.

Schanz, T. and Vermeer, P.A. (1997). On the Stiffness of Sands. Proceedings Symposium on Pre-failure Deformation Behaviour of Geomaterials, ICE, London, 383-387.

Schmertmann, J.H., Baker, W., Gupta, R. and Kessler, K. (1986). CPT/DMT Quality control of ground modification at a power plant. Proceedings of In Situ '86, Conference on Use of In Situ Tests in Geotechnical Engineering, Virginia Tech, Blacksburg, ASCE Geot. Special Publ. No. 6, 985-1001.

Schnaid, F. (2009). In Situ Testing in Geomechanics - the main tests. Taylor \& Francis Group, London.

TC16 (2001). The Flat Dilatometer Test (DMT) in Soil Investigations. A Report by the ISSMGE Committee TC16. Reprinted in Proceedings of the $2^{\text {nd }}$ International Conference on the Flat Dilatometer, 2006, Washington D.C., 7-48.

Totani, G., Calabrese, M., Marchetti, S. and Monaco, P. (1997). Use of in situ flat dilatometer (DMT) for ground characterization in the stability analysis of slopes. Proceedings XIV ICSMFE, Hamburg, vol. 1, 607-610.

Totani, G., Calabrese, M. and Monaco, P. (1998). In situ determination of $\mathrm{C}_{\mathrm{h}}$ by Flat Dilatometer (DMT). Proceedings of the First International Conference on Site Characterization ISC '98, Atlanta, Georgia, USA, 883-888.

Totani, G., Monaco, P., Marchetti, S. and Marchetti, D. (2009). $\mathrm{V}_{\mathrm{S}}$ measurements by Seismic Dilatometer (SDMT) in non penetrable soils. Proceedings $17^{\text {th }}$ ICSMGE, Alexandria, Egypt, 2, 977-980.

Yu, H.S. (2004). In situ soil testing: from mechanics to interpretation. Proceedings $2^{\text {nd }}$ International Conference on Site Characterization, ISC-2, Porto. vol. 1, 3-38. 